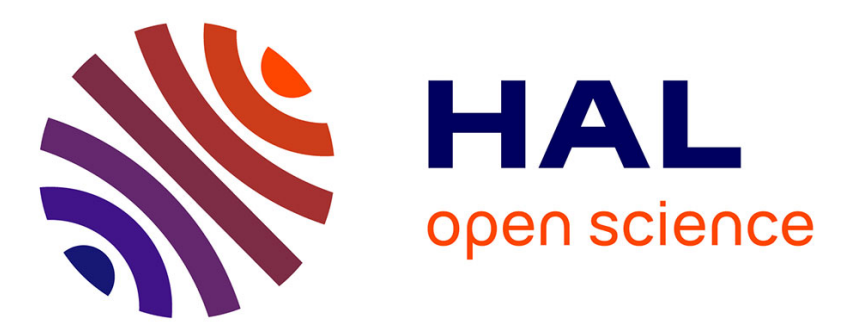

\title{
Essai de modélisation de l'utilisation de l'énergie dans les racines. Application à l'effet de la température racinaire chez des plantules de deux hybrides de maïs Jean-Sylvain Frossard, Maurice M. Crocombette
}

\section{- To cite this version:}

Jean-Sylvain Frossard, Maurice M. Crocombette. Essai de modélisation de l'utilisation de l'énergie dans les racines. Application à l'effet de la température racinaire chez des plantules de deux hybrides de maïs. Agronomie, 1986, 6 (4), pp.401-404. hal-00884891

\section{HAL Id: hal-00884891 \\ https://hal.science/hal-00884891}

Submitted on 1 Jan 1986

HAL is a multi-disciplinary open access archive for the deposit and dissemination of scientific research documents, whether they are published or not. The documents may come from teaching and research institutions in France or abroad, or from public or private research centers.
L'archive ouverte pluridisciplinaire HAL, est destinée au dépôt et à la diffusion de documents scientifiques de niveau recherche, publiés ou non, émanant des établissements d'enseignement et de recherche français ou étrangers, des laboratoires publics ou privés. 


\title{
Essai de modélisation de l'utilisation de l'éner- gie dans les racines. Application à l'effet de la température racinaire chez des plantules de deux hybrides de maïs
}

\author{
Jean-Sylvain FROSSARD \\ avec la collaboration technique de Maurice CrocombetTE \\ I.N.R.A., Laboratoire de Bioclimatologie, Domaine de Crouelle, F 63039 Clermont-Ferrand
}

RÉSUMÉ

\begin{abstract}
En employant les principes de définition de respiration de croissance et de respiration d'entretien utilisés depuis un certain temps pour la plante entière, on développe un modèle d'utilisation de l'énergie disponible dans les racines. Cette modélisation est utilisée pour qualifier l'effet de la température racinaire sur la répartition de l'énergie dans les racines de jeunes plants de maïs âgés de $14 \mathrm{j}$, pour un hybride de type denté et pour un hybride de type corné. Les résultats montrent qu'une température inférieure à $15^{\circ} \mathrm{C}$ conduit à l'accumulation de matière sèche non structurale chez les 2 hybrides. Cette accumulation est plus forte chez l'hybride denté que chez l'hybride corné. En outre la respiration d'entretien est 2 fois plus importante chez le corné que chez le denté. Malgré les limites de la modélisation liées à la définition de la respiration d'entretien et de croissance, on en conclut que la tolérance au froid est liée, au niveau des racines, à la possibilité de consacrer une grande partie de l'énergie disponible à la croissance (accumulation de matière sèche structurale) même à des températures peu élevées $\left(10^{\circ} \mathrm{C}\right)$.
\end{abstract}

Mots clés additionnels : Tolérance au froid, respiration de croissance, respiration d'entretien.

A tentative model of energy use in roots and its application in assessing the effect of root temperature on seedlings of two maize hybrids. Using the definitions of growth respiration and maintenance respiration proposed by THORNLEY (1970) and MC
CREE (1974) for the whole plant, we have developed a root system model, based on the proposals of SZANIAWSKI
(1981). This was used to study the effect of root temperature on energy partition between growth and respiration
in roots of 14-day old maize plants of two grain types (flint and dent). Growth analysis and respiration
measurements revealed that growth efficiency of the two genotypes were the same. However, the maintenance
coefficient was two times greater for the flint type than for the dent type. In both, lowering root temperature
(from $20^{\circ} \mathrm{C}$ to $10^{\circ} \mathrm{C}$ ) induced non-structural dry matter accumulation, which was higher for the dent type. In
spite of limitations arising from the difficulty of correctly defining maintenance and growth respiration
(discussed in the text), it was concluded that the differences in low root temperature adaptation between the
iwo genolypes mainly resided, for the root system, in limitation of non-siructural dry matter accumulation and
high maintenance coefficient.

Additional key works : Modelling, growth respiration, maintenance respiration, temperature adaptation.

\section{INTRODUCTION}

Lors d'une précédente étude, nous avons souligné que la température du milieu modifiait profondément la respiration racinaire et la croissance en matière sèche de plantules de deux hybrides de maïs (FrosSARD, 1985b) : un abaissement de la température pro- voque une diminution de la respiration et une augmentation de l'accumulation de matière sèche. En outre, à la suite de nombreux auteurs, nous avons observé des différences systématiques de comportement entre l'hybride de type denté (sensible au froid) et l'hybride de type corné (tolérant aux températures basses). 
A partir de la modélisation de la respiration développée par certains auteurs pour la plante entière (répartition entre respiration d'entretien et respiration de croissance) et de son application aux racines déjà proposée par ailleurs (SZANIAWSKI, 1981), d'une part, et des mesures réalisées dans l'étude citée ci-dessus, d'autre part, on se propose de développer une modélisation de l'effet de la température du milieu sur l'utilisation de l'énergie dans les racines des deux hybrides.

\section{PRINCIPES DE LA MÉTHODE}

\section{A. Equations}

D'après les critères définis par THORNLEY (1970, 1977) et les définitions rapportées par RUGET (1981) pour la plante entière, on peut écrire d'une façon générale :

$$
\begin{aligned}
& \mathrm{Rt}=\mathrm{Rc}+\mathrm{Rm} \\
& \mathrm{Rt}=\mathrm{k} \cdot \Delta \mathrm{Ms}+\mathrm{mM}
\end{aligned}
$$$$
\text { et }
$$

\section{TABLEAU 1}

Signification des symboles utilisés ( $m, R c, R m, R t$ et $Y g$ sont exprimés en équivalents de matière sèche).

Meaning of symbols $\left(m, R c, R m, R l\right.$ and $Y_{g}$ in dry matrer equaira-

\begin{tabular}{|c|c|c|}
\hline Symboles & Définition & Unités \\
\hline$\Delta \mathrm{M}$ & $\begin{array}{l}\text { accumulation totale de matière } \\
\text { sèche } \\
\text { total dry matter accumulation }\end{array}$ & $m g . j^{-1}$ \\
\hline$\Delta \mathrm{Mns}$ & $\begin{array}{l}\text { accumulation de matière sèche } \\
\text { non structurale } \\
\text { non structural dry matter } \\
\text { accumulation }\end{array}$ & $m g . j^{-1}$ \\
\hline$\Delta \mathrm{Ms}$ & $\begin{array}{l}\text { accumulation de matière sèche } \\
\text { structurale } \\
\text { structural dry matter accu- } \\
\text { mulation }\end{array}$ & $\mathrm{mg} \cdot \mathrm{j}^{-1}$ \\
\hline I.R. & $\begin{array}{l}\text { intensité respiratoire } \\
\text { respiration rate }\end{array}$ & $\mathrm{mg}_{\mathrm{O}_{2}} \cdot \mathrm{h}^{-1} \cdot \mathrm{g}_{\mathrm{MF}}$ \\
\hline $\mathrm{m}$ & $\begin{array}{l}\text { coefficient d'entretien } \\
\text { maintenance coefficient }\end{array}$ & $m g . m g^{-1} \cdot j^{-1}$ \\
\hline $\mathbf{M}$ & $\begin{array}{l}\text { poids de matière sèche } \\
\text { dry matter weight }\end{array}$ & $\mathrm{mg}$ \\
\hline $\mathrm{MF}$ & $\begin{array}{l}\text { poids de matière fraîche } \\
\text { fresh matter weight }\end{array}$ & $\mathrm{g}$ \\
\hline $\mathrm{Rc}$ & $\begin{array}{l}\text { respiration de croissance } \\
\text { growth respiration }\end{array}$ & $m g \cdot j^{-1}$ \\
\hline $\mathrm{Rm}$ & $\begin{array}{l}\text { respiration d'entretien } \\
\text { maintenance respiration }\end{array}$ & $m g . j^{-1}$ \\
\hline Rt & $\begin{array}{l}\text { respiration totale } \\
\text { total respiration }\end{array}$ & $m g . j^{-1}$ \\
\hline $\mathrm{Yg}$ & $\begin{array}{l}\text { efficience de la croissance } \\
\text { growth efficiency }\end{array}$ & $m g \cdot g^{-1}$ \\
\hline $\begin{array}{l}\Delta \mathrm{M}_{\mathrm{r}}, \Delta \mathrm{Mnsr} \\
\ldots, \mathrm{Yg}_{\mathrm{r}}\end{array}$ & $\begin{array}{l}\text { mêmes paramètres que } \\
\text { ci-dessus pour les racines } \\
\text { the same for the roots }\end{array}$ & \\
\hline
\end{tabular}
lents). avec

$\mathrm{k}=\mathrm{Rc} / \Delta \mathrm{Ms}$ et $\mathrm{m}=\mathrm{M} / \mathrm{Rm}$.

L'efficience de croissance Yg est définie de la façon suivante :

$\mathrm{Yg}=\Delta \mathrm{Ms} /(\Delta \mathrm{Ms}+\mathrm{Rc})$

Autrement dit :

$\Delta \mathrm{Ms}=\mathrm{Rc} . \mathrm{Yg} /(1-\mathrm{Yg})$

En suivant les conclusions de THORNLEY (1982), on suppose que, s'il y a stockage, cette composante n'entraîne aucun coût respiratoire.

Suivant la méthode utilisée par Mc CREE (1974) et son application proposée pour les racines par SZANIAWSKI (1981), on peut considérer que les équations (1) et (2) sont aussi valables pour ces organes. En outre, d'après les résultats de Mc CREE (1974, 1983), les calculs de PENNING DE VRIES et al. (1979) pour la plante entière et ceux de SZANIAWSKI \& KIELKIEWICZ (1982) obtenus pour les parties aériennes et les racines, on peut admettre que :

- Yg ne dépend pas de la température,

- le $Q_{10}$ de $\mathrm{m}$ est égal à 2 , ce qui équivaut à écrire que, à une température $\mathrm{T}, \mathrm{m}_{\mathrm{T}}=\mathrm{m}_{\mathrm{To}} \cdot \mathrm{e}^{0,069(\mathrm{~T}-\mathrm{To})}(5)$.

Les paramètres relatifs aux racines sont notés $X_{r}$, par exemple $\mathrm{Rt}_{\mathrm{r}}$ ou $\mathrm{M}_{\mathrm{r}}$.

Donc, pour les racines, à une température donnée To, si on mesure $\mathrm{Rt}_{r}, \mathrm{Rm}_{\mathrm{r}}, \Delta \mathrm{Ms}_{\mathrm{r}}$, on peut calculer $\mathrm{Yg}_{\mathrm{r}}$ et $m_{r}$ à partir des formules (2) et (3).

Remarquons qu'il faut que, à To, les conditions soient telles que $\Delta \mathrm{M}_{\mathrm{r}}$ (mesuré) $=\Delta \mathrm{Ms}_{\mathrm{r}}$.

Ceci implique, à une autre température $\mathrm{T}$, un certain nombre de conditions :

$-\Delta \mathrm{M}_{\mathrm{r}}$ ne peut être inférieur à $\Delta \mathrm{Ms}_{\mathrm{r}}$.

- Si $\Delta \mathrm{M}_{\mathrm{r}}$ est supérieur à $\Delta \mathrm{Ms}_{\mathrm{r}}$ calculé à partir de la formule (3), on admet qu'il y a accumulation de matière sèche non structurale, notée $\Delta \mathrm{Mns}_{\mathrm{r}}$ et calculée de la façon suivante :

$\Delta \mathrm{Mns}_{\mathrm{r}}=\Delta \mathrm{M}_{\mathrm{r}}-\left(\mathrm{Rt}_{\mathrm{r}}-\mathrm{m}_{\mathrm{r}} \mathrm{M}_{\mathrm{r}}\right) \cdot \mathrm{Yg}_{\mathrm{r}} /\left(1-\mathrm{Yg}_{\mathrm{r}}\right) \cdot(6)$

L'équation (6) correspond, pour les racines, aux propositions avancées par THORNLEY (1977) pour la plante entière à propos de l'existence de deux sortes de matière sèche, l'une dégradable (et pouvant donc être réutilisée pour la croissance), l'autre non dégradable correspondant à la croissance "vraie " (à laquelle s'applique le calcul de Yg).

- Avant la mesure, il n'y a pas eu d'accumulation de matière non structurale (ce qui signifie, entre autres, que les plantes ont été élevées à la température To avant la mesure).

Ainsi, pour une gamme de températures comprises dans le domaine de validité des propositions formulées ci-dessus (entre 5 et $35^{\circ} \mathrm{C}$ ), à partir de mesures directes de $M_{r}, \Delta M_{r}, R_{r}$ et du calcul de $m_{r}$, on peut déduire les valeurs de $\mathrm{Rc}_{\mathrm{r}}, \mathrm{Rm}_{\mathrm{r}}, \Delta \mathrm{Ms}_{\mathrm{r}}$ et $\Delta \mathrm{Mns}_{\mathrm{r}}$.

Quel que soit l'intervalle de temps de mesure, l'énergie reçue par le système racinaire (ou énergie disponible pour les racines) s'écrit :

$\mathrm{E}_{\mathrm{r}}=\mathrm{Rt}_{\mathrm{r}}+\Delta \mathrm{M}_{\mathrm{r}}$.

A partir de cette expression, on peut calculer $P($.$) la$ proportion d'énergie utilisée lors de la respiration (production d'énergie directement utilisable pour les diverses fonctions du système racinaire), pour la croissance au sens strict $\left(\Delta \mathrm{Ms}_{\mathrm{r}}\right)$, ou pour tout autre paramètre de la façon suivante : $P\left(X_{r}\right)=X_{r} / E_{r}, X_{r}$ étant le paramètre considéré. 
Pour passer d'une équation à une autre, il faut que tous les termes soient dans des unités homogènes. Pour répondre à cette condition, toutes les valeurs d'accroissement de matière, de respiration ou de matière sèche en présence sont converties en équivalents de matière sèche, à partir de l'équation générale ci-dessous, proposée par PENNING DE VRIES et al. (1979) :

$1 \mathrm{~g}$ de $\mathrm{M}$ (et par extension de $\mathrm{Ms}$ ou de $\Delta \mathrm{Ms}$ ) $\approx 0,68 \mathrm{~g}$ de $\mathrm{CO}_{2}$ rejeté.

Considérant que l'on se trouve toujours en conditions normoxiques, le quotient respiratoire est voisin de l'unité ; ce qui équivaut à dire que $\mathrm{V}\left(\mathrm{CO}_{2}\right)=\mathrm{V}\left(\mathrm{O}_{2}\right)$, alors :

$1 \mathrm{~g}$ de $\mathrm{O}_{2}$ absorbé pour la respiration $\approx 2,022 \mathrm{~g}$ de $\mathrm{M}$ (et par extension de Ms ou de $\Delta \mathrm{Ms}$ ).

\section{B. Principes de la mesure de $\mathrm{Yg}_{\mathbf{r}}$ et de $\mathrm{m}_{\mathrm{r}}$}

Ils découlent des considérations du paragraphe précédent.

Pour calculer $\mathrm{Yg}_{\mathrm{r}}$, il faut disposer de la valeur de $m_{r}$.

Pour mesurer $m_{r}$, il faut mesurer $R_{r}$ et $M_{r}$ en l'absence de croissance en matière sèche. Pour ce faire, on utilise la méthode développée par Mc CREE (1974) pour la plante entière :

$\mathrm{m}=\mathrm{Rt} / \mathrm{M}$ après $36 \mathrm{~h}$ d'obscurité.

Dans une étude précédente (FroSSARD, 1985a), nous avons relevé que, après $36 \mathrm{~h}$ d'obscurité, chez le tournesol, la croissance des racines est complètement arrêtée et que leur respiration présente une stabilisation relative à un niveau nettement plus bas que lorsque les parties aériennes sont soumises à un rythme d'éclairement. En outre à ce moment-là, la respiration s'appuie exclusivement sur un (ou des) substrat(s) contenu(s) dans les racines; ce(s) substrat(s) ne dépend(ent) pas de la photosynthèse du jour précédent comme l'avaient montré auparavant PEARSON (1974) sur la fève et, indirectement, FARRAR (1980b) sur l'orge.

$\mathrm{Si}$ on applique ces principes aux racines de maïs, cela suppose de mesurer à une température donnée :

- $\mathrm{Rt}_{\mathrm{r}}, \Delta \mathrm{Ms}_{\mathrm{r}}$, en conditions normales d'éclairement de croissance, c'est-à-dire lors d'une séquence lumière/obscurité ou, plus précisément, lors d'une séquence photophase/scotophase ;

- $R t_{r}$ (qui est égale à $R m_{r}$ à ce moment-là) et $M_{r}$, après $36 \mathrm{~h}$ d'obscurité.

Les valeurs ainsi obtenues permettent de calculer $\mathrm{Yg}_{\mathrm{r}}$ et $\mathrm{m}_{\mathrm{r}}$ au moyen des équations (2) et (3).

En l'absence d'information sur l'évolution de la croissance des racines de maïs quand la partie aérienne est plongée dans l'obscurité au-delà de la scotophase de durée habituelle, l'application des principes avancés ci-dessus rend nécessaire un contrôle de la croissance des racines et des parties aériennes dans ces conditions.

En outre, on sait, d'après les travaux de HANSEN \& JENSEN (1977) et de HANSEN $(1979,1980)$, que $\mathrm{Rc}_{\mathrm{r}}$ et $\mathrm{Rm}_{\mathrm{r}}$ sont susceptibles de varier en fonction du type d'alimentation en azote (nitrique ou ammoniacal), toutes choses égales par ailleurs (taille de la plante, température, etc.), ce qui impose un contrôle rigoureux des conditions d'alimentation minérale.

\section{MATÉRIEL VÉGÉTAL ET TECHNIQUES EXPÉRIMENTALES}

\section{A. Matériel végétal}

Il se compose de plantules issues de 2 hybrides simples :

- F7 × F2 de type corné, tolérant aux températures basses au printemps en conditions normales de culture, au champ ;

- WH $\times$ WJ de type denté, sensible au froid printanier.

Les plantes sont cultivées sur solution nutritive convenablement aérée. Les conditions de cultures sont similaires à celles décrites dans un article précédent (FROSSARD, 1985b).

Les températures racinaires sont : 10, 15, 20 (témoin, température identique à celle de la culture) et $25{ }^{\circ} \mathrm{C}$.

Pendant la culture et lors des mesures de respiration et de croissance, la solution nutritive est renouvelée totalement chaque semaine pour éviter toute carence minérale.

Les plantes sont soumises aux différents traitements à l'âge de $14 \mathrm{j}$.

\section{B. Mesure et calcul de $\mathrm{Yg}_{\mathrm{r}}$ et de $\mathrm{m}_{\mathrm{r}}$}

La culture des plantes est réalisée à $20^{\circ} \mathrm{C}$ constants. En suivant les principes exposés précédemment lors de la formulation de l'équation (6), c'est à cette température que seront calculés $\mathrm{Yg}_{\mathrm{r}}$ et $\mathrm{m}_{\mathrm{r}(20)}$. D'après l'équation (5), à toute température $\mathrm{T}, \mathrm{m}_{\mathrm{r}(\mathrm{T})}$ est tel que :

$$
\mathrm{m}_{\mathrm{r}(\mathrm{T})}=\mathrm{m}_{\mathrm{r}(20)} \cdot \mathrm{e}^{0,069(\mathrm{~T}-20)} \text {. }
$$

Pour des raisons méthodologiques, les accroissements de matière sèche et la respiration racinaire n'ont pu être mesurés sur les mêmes individus (cf. § D et $\S \mathrm{E}$ pour les méthodes).

\section{Calcul de l'utilisation de $E_{r}$ à différentes tempéra- tures}

$E_{r}$ et $P\left(X_{r}\right)$ sont calculés à partir de l'équation (7) et des valeurs de $X_{r}$ calculées ou mesurées.

Pour les autres températures expérimentales, 10, 15 et $25{ }^{\circ} \mathrm{C}$, on mesure seulement $\Delta \mathrm{M}_{\mathrm{r}}$ et $\mathrm{Rt}_{\mathrm{r}}$. Les valeurs de $\Delta \mathrm{Ms}_{\mathrm{r}}, \Delta \mathrm{Mns}_{\mathrm{r}}$ et de $\mathrm{Rm}_{\mathrm{r}}$ sont calculées à partir des équations (1), (4) et (6).

\section{Mesure de la respiration racinaire}

La respiration est appréciée par l'absorption d'oxygène mesurée au moyen d'une électrode polarographique ; les mesures sont réalisées en continu avec un respiromètre automatique décrit par ailleurs (FROSSARD, 1976). 
Le protocole de mesure est le même que celui décrit précédemment (FrOSSARD, 1985b) pour les températures de 15 et $25^{\circ} \mathrm{C}$.

Pour les températures de 10 et $20^{\circ} \mathrm{C}$, on mesure la respiration racinaire pendant une succession de 2 scotophases et d'une photophase, puis en obscurité continue pendant au moins $36 \mathrm{~h}$. Le nombre d'individus par traitement (température $\times$ génotype) est de 4 . Le coefficient de variation des moyennes est de 15 p. 100 environ.

\section{E. Analyse de la croissance}

Le protocole est identique à celui employé dans une étude précédente (FrOSSARD, 1985b). Il y a 8 répétitions par traitement.

Les valeurs présentées dans les tableaux de résultats proviennent de l'étude citée.

La teneur en azote organique des différentes parties de la plante est appréciée par la différence entre la teneur en azote total mesurée par la méthode Kjeldahl et la teneur en azote nitrique mesurée par ionométrie sur un éluat de la matière sèche.

\section{RÉSULTATS ET DISCUSSION}

\section{A. Estimation de $\mathbf{Y g}_{\mathrm{r}}$ et de $\mathrm{m}_{\mathrm{r}}$}

D'après les principes développés dans le chapitre II, on mesure $\mathrm{Rt}_{\mathrm{r}}$ et $\mathrm{m}_{\mathrm{r}}$ en l'absence d'augmentation de matière sèche. Les résultats présentés sur la figure 1
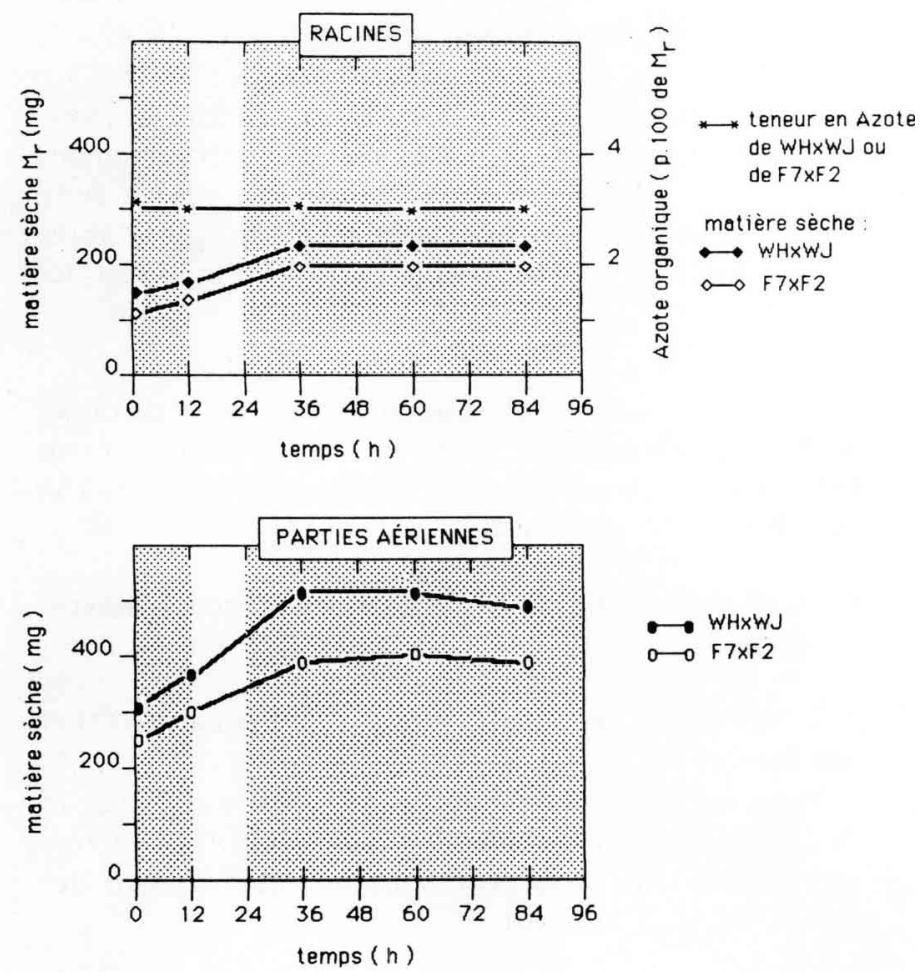

Figure 1

Accroissement de matière sèche des racines et des parties aériennes et variation du taux d'azote organique des racines au cours du temps, à $20^{\circ} \mathrm{C}$, lors d'un cycle lumiere-obscurité et lors d'une obscurité prolongée.

Dry matter growth of roots and shoots and organic nitrogen ratio of roots, at $20^{\circ} \mathrm{C}$, with a normal day-night period (from 0 to $36 \mathrm{~h}$ ) or extended darkness (from 36 to $96 \mathrm{~h}$ ). montrent que cette condition est réalisée après $24 \mathrm{~h}$ d'obscurité à $20^{\circ} \mathrm{C}$. Dans ces conditions, la respiration racinaire évolue lentement à des valeurs nettement inférieures à celles mesurées lors d'un cycle lumière-obscurité (fig. 2). Enfin, comme il n'y a pas altération de la teneur en azote organique des racines (fig. 1), il est vraisemblable que l'intégrité fonctionnelle des racines soit maintenue.

L'ensemble de ces résultats permet d'avancer que $\mathrm{Rt}_{\mathrm{r}}$ mesurée après $24 \mathrm{~h}$ d'obscurité est bien assimilable à $\mathrm{Rm}_{\mathrm{r}}$. A partir des formules (2) et (3) appliquées aux racines, on peut alors calculer les valeurs de $\mathrm{Yg}_{\mathrm{r}}$ et de $\mathrm{m}_{\mathrm{r}(20)}$. Celles-ci sont présentées au tableau 2 .

L'efficience de croissance est sensiblement la même pour les 2 hybrides. Elle est proche des valeurs trouvées par ailleurs pour le tournesol (SZANIAWSKI \& KIELKIEWICZ, 1982) ou pour le ray-grass italien (HANSEN \& JENSEN, 1977). Par contre, le coefficient d'entretien de l'hybride tolérant au froid est 2 fois plus élevé que celui de l'hybride peu tolérant.
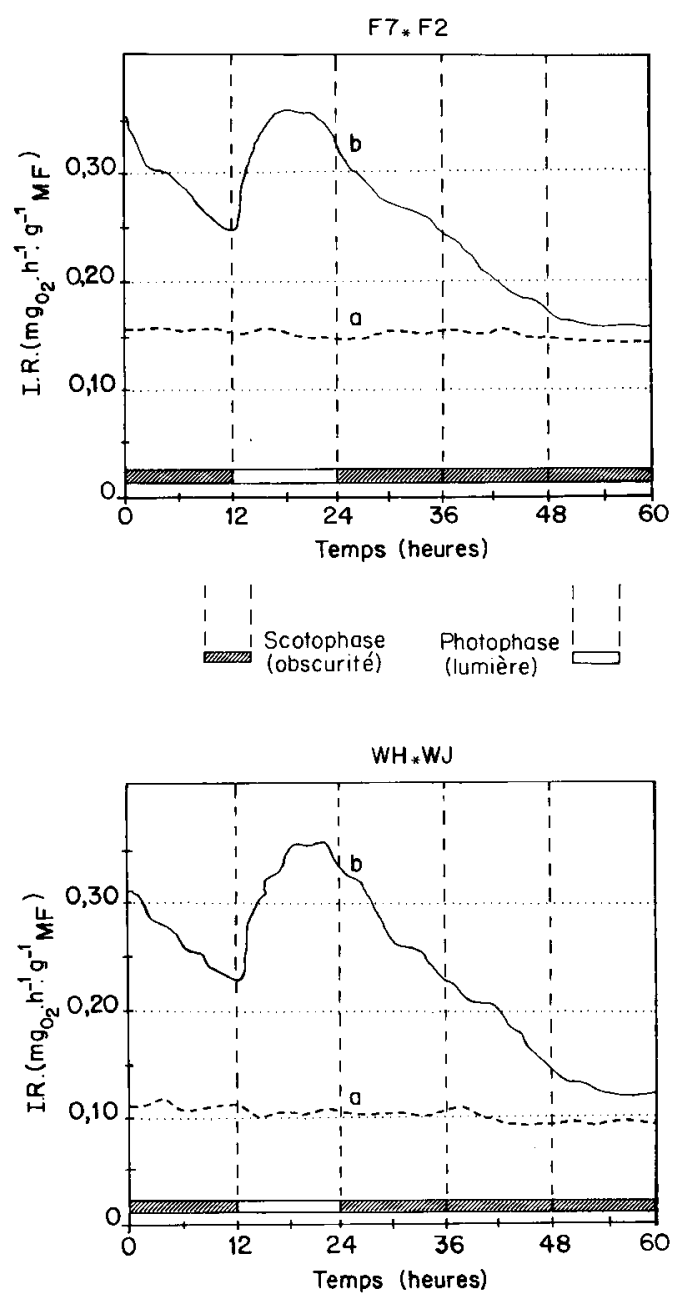

Figure 2

Evolution de l'intensité respiratoire racinaire au cours du temps, à $10^{\circ} \mathrm{C}$ (a) et à $20^{\circ} \mathrm{C}(\mathrm{b})$, lors d'un cycle lumière-obscurité et lors d'une obscurité prolongée. (L'humidité pondérale des racines est sensiblement constante tout au long des expériences avec les valeurs suivantes à $10{ }^{\circ} \mathrm{C}$ et $20^{\circ} \mathrm{C}$ : pour $W H \times W J, 5,5$ et $18,0 \mathrm{p} .100$, pour $F 7 \times F 2,14,2$ et $15,6 \mathrm{p}$. 100).

Root respiration rate at different root temperatures: (a) $10{ }^{\circ} \mathrm{C}$, (b) $20{ }^{\circ} \mathrm{C}$, with a normal day-night period (from 0 to $36 \mathrm{~h}$ ) or extended darkness (from 36 to $84 \mathrm{~h}$ ). (The water content of roots may be considered as constant at $10^{\circ} \mathrm{C}$ and $20^{\circ} \mathrm{C}:$ for $W H \times W J, 5.5$ and $18.0 \%$, for $F 7 \times F 2,14.2$ and $15.6 \%)$. 
TABLEAU 2

Efficience de croissance et coefficient d'entretien pour les deux hybrides avec intervalle de confiance à $5 \%$.

Growth efficiency and maintenance coefficient for the two hybrids (5\% confidence interval).

\begin{tabular}{lrr}
\hline \hline Hybride & \multicolumn{1}{c}{$\mathrm{Yg}_{\mathrm{r}}$} & $\mathrm{m}_{\mathrm{r}(20)}$ \\
\hline $\mathrm{F} 7 \times \mathrm{F} 2$ & 0,666 & 0,188 \\
& $\pm 0,183$ & $\pm 0,051$ \\
\hline WH $\times$ WJ & 0,643 & 0,091 \\
& $\pm 0,152$ & $\pm 0,027$ \\
\hline \hline
\end{tabular}

Bien que la présentation des résultats et la discussion puissent paraître cohérents, il convient d'émettre quelques réserves quant à une généralisation trop rapide de ce type de résultats, pour plusieurs raisons.

En premier lieu, nous avons considéré jusqu'à présent, à la suite de nombreux auteurs, que la respiration avait toujours une finalité énergétique. Or il existe un phénomène respiratoire (absorption de $\mathrm{O}_{2}$ et rejet de $\mathrm{CO}_{2}$ associés à une dégradation de glucides) qui ne produit pas d'énergie pour l'organe (LAMBERS, 1982 et travaux antérieurs). L'importance de cette respiration non-phosphorylante est très variable suivant les organes, l'âge du végétal, les conditions climatiques et les espèces (LAMBERS, 1982).

En second lieu, si $\mathrm{Rm}_{\mathrm{r}}$ paraît assez facile à définir, il n'en va pas de même de $\mathrm{Rc}_{\mathrm{r}}$. En effet, l'énergie libérée par $\mathbf{R c}_{\mathrm{r}}$ est certes utilisée pour la croissance des racines mais aussi pour des fonctions servant à la croissance des autres organes de la plante. Ainsi en est-il de l'absorption minérale dont le coût énergétique peut représenter jusqu'à 30 p. 100 de $R_{c_{r}}$ chez le maïs (VEEN, 1980).
Enfin, comme l'ont souligné LAMBERS et al. (1983) dans une revue bibliographique, de nombreux autres éléments, tels que la composition biochimique ou la demande " externe " des organes pour leur croissance peuvent modifier la signification de $R c_{r}$ et de $R m_{r}$ et donc de $\mathrm{Yg}_{\mathrm{r}}$ et de $\mathrm{m}_{\mathrm{r}}$.

\section{B. Effet de la température sur l'utilisation de l'éner- gie disponible}

La croissance et la respiration aux différentes températures ainsi que la répartition de l'énergie disponible entre différents " types » de respiration et croissance en matière sèche est présentée au tableau 3 .

Avant de discuter ces résultats, il convient de revenir sur les cinétiques de respiration présentées à la figure 2 . A $10^{\circ} \mathrm{C}$, il n'y a pas de différence entre la scotophase (obscurité de durée habituelle, $12 \mathrm{~h}$ ) et l'obscurité prolongée. Il est donc difficile de définir une respiration d'entretien dans les mêmes conditions qu'à $20^{\circ} \mathrm{C}$.

$\mathrm{Si}$ on considère que $\mathrm{Rt}_{\mathrm{r}}$ au cours d'un cycle lumièreobscurité ou photophase-scotophase $\left(\mathrm{Rt}_{\mathrm{r}}(\mathrm{J} / \mathrm{N})\right)$ est égale à $\mathrm{Rm}_{\mathrm{r}}$, ceci équivaut à dire qu'il n'y a pas de croissance pendant la photophase et la scotophase. Or, l'analyse de croissance présentée au tableau 3 révèle qu'il y a accroissement de matière sèche dans cet intervalle de temps. Cette proposition est donc à rejeter. En outre, nous avons remarqué que, dans l'intervalle de temps des mesures d'accroissement, la photosynthèse nette n'était pas modifiée par la température racinaire (FROSSARD, 1985b). Donc, si $\mathrm{Rt}_{\mathrm{r}}(\mathrm{J} / \mathrm{N})$ est égale à $\mathrm{Rt}_{\mathrm{r}}$ après $24 \mathrm{~h}$ d'obscurité, c'est probablement parce qu'il y a eu suffisamment d'assi-

TABLEAU 3

Respiration d'entretien, respiration de croissance, croissance en matière sèche et taux d'utilisation de l'énergie disponible pour les deux hybrides: (a) pour F7 $\times F 2$; (b) pour $W H \times W J$.

Maintenance respiration, growth respiration, dry matter growth and energy utilization ratios for the two hybrids : (a) for $F 7 \times F 2$; (b) for $W H \times W J$.

Tableau $3 a$

\begin{tabular}{lcccc}
\hline \hline Température & $10{ }^{\circ} \mathrm{C}$ & $15{ }^{\circ} \mathrm{C}$ & $20{ }^{\circ} \mathrm{C}$ & $25{ }^{\circ} \mathrm{C}$ \\
\hline $\mathrm{M}_{\mathrm{r}}$ & 110 & 110 & 110 & 110 \\
\hline$\Delta \mathrm{M}_{\mathrm{r}}$ & 40,0 & 30,0 & 25,6 & 26,4 \\
\cline { 2 - 5 } $\mathrm{M}_{\mathrm{r}}+\Delta \mathrm{M}_{\mathrm{r}}$ & 150,0 & 140,0 & 135,6 & 136,4 \\
\cline { 2 - 5 } $\mathrm{Rt}_{\mathrm{r}}$ & 18,8 & 28,5 & 33,5 & 42,4 \\
\hline $\mathrm{Rm}_{\mathrm{r}}$ & 10,4 & 14,7 & 20,7 & 29,2 \\
\hline $\mathrm{Rc}_{\mathrm{r}}$ & 8,4 & 13,9 & 12,8 & 13,1 \\
\hline$\Delta \mathrm{Ms}_{\mathrm{r}}$ & 16,8 & 27,7 & 25,6 & 26,4 \\
\hline$\Delta \mathrm{Mns}_{\mathrm{r}}$ & 23,2 & 2,3 & 0 & 0 \\
\hline $\mathrm{E}_{\mathrm{r}}$ & 58,8 & 58,5 & 59,1 & 68,8 \\
\hline $\mathrm{P}\left(\mathrm{Rc}_{\mathrm{r}}+\Delta \mathrm{M}_{\mathrm{r}}\right)$ & 82,3 & 75,0 & 65,0 & 57,5 \\
\hline $\mathrm{P}\left(\mathrm{Rm}_{\mathrm{r}}\right)$ & 17,7 & 25,0 & 35,0 & 42,5 \\
\hline \hline
\end{tabular}

Tableau $3 b$

\begin{tabular}{lcccc}
\hline \hline Température & $10{ }^{\circ} \mathrm{C}$ & $15{ }^{\circ} \mathrm{C}$ & $20{ }^{\circ} \mathrm{C}$ & $25{ }^{\circ} \mathrm{C}$ \\
\hline $\mathrm{M}_{\mathrm{r}}$ & 140 & 140 & 140 & 140 \\
\hline $\mathrm{M}_{\mathrm{r}}$ & 130,0 & 80,0 & 73,0 & 71,0 \\
\hline $\mathrm{M}_{\mathrm{r}}+\Delta \mathrm{M}_{\mathrm{r}}$ & 270 & 220 & 213 & 211 \\
\hline $\mathrm{Rt}_{\mathrm{r}}$ & 20,1 & 36,1 & 53,4 & 57,7 \\
\hline $\mathrm{Rm}_{\mathrm{r}}$ & 6,4 & 9,1 & 12,8 & 18,1 \\
\hline $\mathrm{Rc}_{\mathrm{r}}$ & 13,7 & 27,0 & 40,6 & 39,6 \\
\hline$\Delta \mathrm{Ms}_{\mathrm{r}}$ & 24,6 & 48,6 & 73,0 & 71,0 \\
\hline$\Delta \mathrm{Mns}_{\mathrm{r}}$ & 105,4 & 31,4 & 0 & 0 \\
\hline $\mathrm{E}_{\mathrm{r}}$ & 150,1 & 116,1 & 126,4 & 128,7 \\
\hline $\mathrm{P}\left(\mathrm{Rc}_{\mathrm{r}}+\Delta \mathrm{M}_{\mathrm{r}}\right)$ & 95,7 & 92,2 & 89,9 & 86,0 \\
\hline $\mathrm{P}\left(\mathrm{Rm}_{\mathrm{r}}\right)$ & 4,3 & 7,8 & 10,1 & 14,0 \\
\hline \hline
\end{tabular}




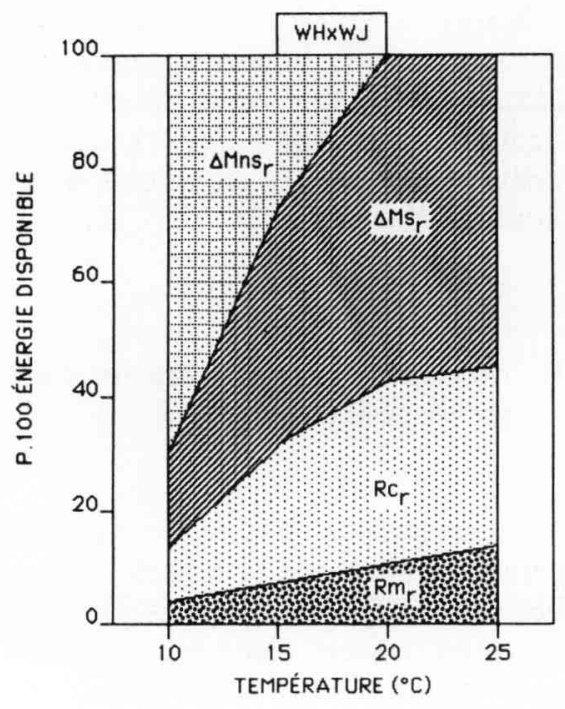

Figure 3

Utilisation de l'énergie disponible dans les racines suivant la température pour les deux hybrides.

milats accumulés avant l'obscurité prolongée. Il paraît illusoire à ce moment-là de chercher à définir $\mathrm{Rm}_{\mathrm{r}}$ $\left(10^{\circ} \mathrm{C}\right)$ autrement que par le calcul en utilisant la formule (5).

L'acceptation de cette proposition conduit aussi à admettre que la matière sèche accumulée lors de la scotophase et de la photophase est en partie composée de matériaux facilement réutilisables pour la respiration. Cette constatation rejoint la formulation de la matière sèche non structurale proposée au chapitre II. Elle est conforme aux observations rapportées par FARRAR (1980a) pour l'orge et par MOLDAU \& SOBER (1981).

La répartition de l'énergie disponible suivant la température, pour les 2 hybrides est représentée sur la figure 3.

On constate que, quel que soit l'hybride, la proportion de matière sèche non structurale augmente quand la température diminue. Cependant, la production de matière sèche structurale est moins affectée par un abaissement de la température chez F7 $\times$ F2 que chez WH $\times$ WJ. A partir des travaux de MOLDAU \& SOBER (1981) qui soulignent que la tolérance aux températures basses est associée à une limitation de l'accumulation de matière sèche non structurale, on peut avancer que l'hybride F7 $\times$ F2 s'adapte plus facilement à un abaissement de température que l'hybride WH $\times$ WJ. Ceci est en accord avec les différences de comportement des 2 hybrides déjà rapportées par ailleurs (FROSSARD, 1985b).

On peut souligner enfin que la part d'énergie disponible consacrée à $\mathrm{Rm}_{\mathrm{r}}$ est beaucoup plus importante chez l'hybride tolérant au froid.

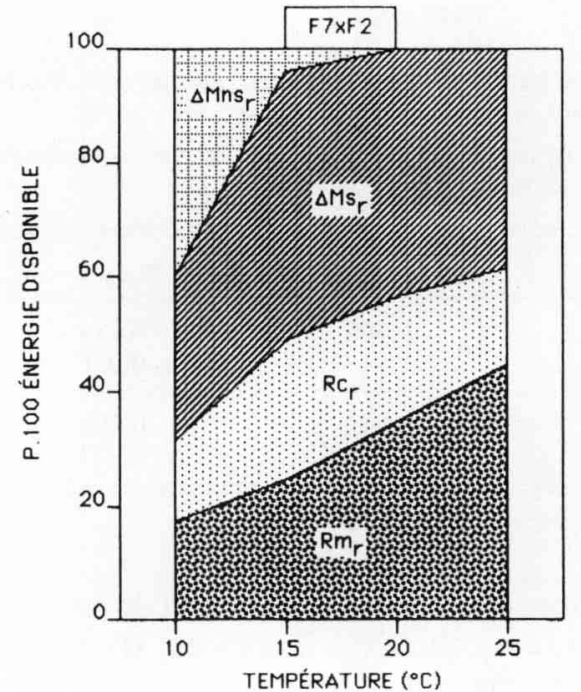

Energy utilization ratios at different root temperatures for the roots of the two hybrids.

\section{CONCLUSION}

Cette étude montre qu'il est possible de modéliser l'utilisation de l'énergie disponible dans les racines en employant des définitions similaires à celles utilisées depuis un certain temps pour la plante entière.

Malgré les réserves que l'on peut faire sur la définition du coefficient de maintenance et, plus particulièrement, sur celle de l'efficience de croissance, cette modélisation permet une représentation beaucoup plus figurative des différences d'utilisation de l'énergie induites par la température dans les racines d'hybrides de mais ayant une tolérance aux températures basses différente. Cette tolérance, déjà expérimentée au champ, est associée à la capacité de consacrer plus d'énergie à l'élaboration de matière sèche structurale à basse température ainsi qu'à un coefficient de maintenance plus élevé.

Les principes développés et leur vérification expérimentale, bien que limitée, pourraient permettre d'affiner le modèle général de la répartition de l'énergie dans la plante proposé par CHRISTIAN \& MILTHORPE (1981).

Il est possible que les résultats obtenus en régime thermique constant le soient aussi en régime fluctuant, comme c'est le cas pour le modèle général développé pour la plante entière (Mc CREE \& AMTHOR, 1982).

L'extension de cette modélisation à tout un cycle de végétation ne peut être envisagée qu'après une large vérification expérimentale. En effet, les travaux de RUGET et al. (1981) ont montré qu'il était très difficile de ne retenir qu'un seul couple de valeurs Yg-m pour tout le cycle de végétation du maïs. 


\section{RÉFÉRENCES BIBLIOGRAPHIQUES}

Christian K. R., Milthorpe F. L., 1981. A systematic approach to the simulation of short-term processes in the plant-environment complex. Plant and Cell Environment, 4, 275-284.

Farrar J. F., 1980a. Allocation of carbon to growth storage and respiration in the vegetative barley plant. Plant and Cell Environment, 3, 97-105.

Farrar J. F,, $1908 \mathrm{~b}$. The pattern of respiration rate in the vegetative barley plant. Ann. Bot., 46, 71-76.

Frossard J. S., 1976. Relations entre l'éclairement des feuilles et l'absorption d'oxygène par les racines chez le tournesol (Helianthus annuus L.). Ann. Agron., 27, 435-445.

Frossard J. S., 1985a. L'éclairement du feuillage facteur de régulation du rythme nycthéméral de la respiration racinaire. Physiol. Vég., 23 (2), 163-173.

Frossard J. S., 1985b. Effet de la température des racines sur la respiration de celles-ci et la croissance de plantules de deux hybrides de maïs. Agronomie, 5 (8), 719-725.

Hansen G. K., 1979. Influence of nitrogen form and nitrogen absence on utilization of assimilates for growth and maintenance in tops and roots of Lolium multiflorum. Physiol. Plant., 46, 165-168.

Hansen G. K., 1980. Diurnal variation of root respiration rates and nitrate uptake as influenced by nitrogen supply. Physiol. Plant., 48, 421-427.

Hansen G. K., Jensen C. R., 1977. Growth and maintenance respiration in whole plants, tops, and roots of Lolium multiflorum. Physiol. Plant., 39, 155-164.

Lambers H., 1982. Cyanide-resistant respiration : a nonphosphorylating electron transport pathway acting as an energy overflow. Physiol. Plant., 55, 478-485.

Lambers H., Szaniawski R. K., Visser R. de, 1983. Respiration for growth, maintenance and ion uptake. An evaluation of concepts, methods, values and their significance. Physiol. Plant., 58, 556-563.

McCree K. J., 1974. Equations for the rate of dark respiration of white clover and grain sorghum, as functions of dry weight, photosynthetic rate, and temperature. Crop Sci., 14, 509-514.

McCree K. J., 1983. Carbon balance as a function of plant size in sorghum plants. Crop Sci., 23, 1173-1177.
McCree K. J., Amthor M. E., 1982. Effects of diurnal variation in temperature on the carbon balances of white clover plants. Crop Sci., 22, 822-827.

Moldau H., Sober J., 1981. Growth rate-reserve content relationship as influenced by irradiance, carbon dioxide concentration, and temperature. Photosynthesis Res., 1, 217-231.

Pearson C. J., 1974. Daily changes in carbon-dioxide exchange and photosynthate translocation of leaves of Vicia faba. Planta, 119 , 59-70.

Penning De Vries F. W. T., Witlage J. M., Kremer D. K., 1979. Rates of respiration and of increase in structural dry matter in young wheat, ryegrass and maize plants in relation to temperature, to water stress and to their sugar content. Ann. Bot., 44, 595-609.

Ruget F., 1981. Respiration de croissance et respiration d'entretien : méthodes de mesure, comparaison des résultats. Agronomie, 1, 601610.

Ruget F., André M., Massimino J., 1981. Evolution de la respiration et croissance, au cours d'un cycle de végétation, de maïs cultivé en chambre de mesure. Physiol. Vég., 19, 277-299.

Szaniawski R. K., 1981. Growth and maintenance respiration of shoot and roots in scots pine seedlings. Z. Pflanzenphysiol., 101, 391-398.

Szaniawski R. K., Kielkiewicz M., 1982. Maintenance and growth respiration in shoots and roots of sunflower plants grown at different root temperature. Physiol. Plant., 54, 500-504.

Thornley J. H. M., 1970. Respiration, growth and maintenance in plants. Nature, 227, 304-305.

Thornley J. H. M., 1977. Growth, maintenance and respiration : a re-interpretation. Ann. Bot., 41, 1197-1203.

Thornley J. H. M., 1982. Interpretation of respiration coefficients. Ann. Bot., 49, 257-259.

Veen B. W., 1980. Energy cost of ion transport, 187-195. In D. W. Rains. R. C. Valsmine \& A. Hollacnder : "Cenenc lenginesering of Usmoregulation. Impact on Plane Productivity for Food, Chemical and Energy ». Plenum Press, New York, ISBN 0306305450. 